\title{
Editorial to the Topical Collection on High Performance Clocks with Special Emphasis on Geodesy and Geophysics and Applications to Other Bodies of the Solar System
}

\author{
Véronique Dehant ${ }^{1} \cdot$ Leonid I. Gurvits ${ }^{2,3}$. \\ Michael Kramer ${ }^{4,5}$ - Ryan Park $^{6}$ - Peter Wolf ${ }^{7}$. \\ John Zarnecki ${ }^{8,9} \cdot$ Rafael Rodrigo 9
}

Received: 15 November 2017 / Accepted: 19 November 2017 / Published online: 21 December 2017

(C) Springer Science+Business Media B.V., part of Springer Nature 2017

\section{Role of Clocks in Fundamental Physics}

As described by one of the papers of this topical collection (Delva et al. 2017), there are two fundamental theories of physics: the Standard Model of particle physics that describes electromagnetism and the (strong and weak) nuclear interactions, and General Relativity that accounts for all gravitational phenomena. The dream of all physicists is to unify these two theories, describing as much as possible of the observed Universe and solving the stillremaining open issues of both theories and observations (e.g. the nature of dark matter and dark energy). Most physicists agree that there is still one theory that remains to be discovered

High Performance Clocks with Special Emphasis on Geodesy and Geophysics and Applications to Other Bodies of the Solar System

Edited by Rafael Rodrigo, Véronique Dehant, Leonid Gurvits, Michael Kramer, Ryan Park, Peter Wolf and John Zarnecki

\footnotetext{
$\bowtie$ V. Dehant

v.dehant@oma.be

1 Royal Observatory of Belgium, Brussels, Belgium

2 Joint Institute for VLBI ERIC (European Research Infrastructure Consortium), Dwingeloo, The Netherlands

3 Department of Astrodynamics and Space Missions, Delft University of Technology, Delt, The Netherlands

4 MPI für Radioastronomy, Bonn, Germany

5 Jodrell Bank Centre for Astrophysics, University of Manchester, Manchester, UK

6 Jet Propulsion Laboratory, Pasadena, USA

7 SYRTE, Observatoire de Paris, PSL Research University, CNRS, Sorbonne Universités, UPMC, Univ. Paris 06, LNE, 75014 Paris, France

8 Open University, London, UK

9 ISSI, Bern, Switzerland
} 
in that respect. Attempts at such a unified theory lead to tiny violations of the basic principles of these theories, in particular of the Einstein Equivalence Principle (i.e., equivalence of gravitational and inertial masses). Delva et al. (2017) review a number of alternative theories and present their pros and cons, as well as the possibilities that can be offered by further more-precise observations, in particular by using high performance clocks. The search for discoveries at the experimental level requires high accuracy measurements that need the use of the outstanding performance offered by a time/frequency metrology. The role of clocks in these observations is fundamental and their use outside the Earth's gravitational field is also fundamental. Clocks on Earth are limited by the knowledge of the local Earth potential and of its fluctuations. As a matter of fact, the SI units themselves rely on a time/frequency realization.

Precise clocks can be used not only to verify the Einstein Equivalence Principle via precise redshift measurements or tests of gravity at Solar System scale. On Earth, the best reference standard clocks are at the $10^{-18}$ precision level and, in space, they are at $10^{-16}$. Such high-performance clocks can be compared over large distances and in varying gravitational fields when used in space, which makes them very valuable for fundamental physics experiments. Such experiments are described in this topical collection by Delva et al. (2017). In particular, ultra-stable microwave or optical clocks in space from the ESA ACES mission are (1) PHARAO, a laser cooled Cesium clock, and (2) SHM, a space hydrogen maser. They are related to clocks on Earth with an improved version of the Microwave link (MWL) as well as an optical link for high performance space-ground clock comparisons. The launch of the ACES/PHARAO platform aboard a Dragon capsule of SpaceX (CRS-13) is scheduled for mid-2018 and its use is foreseen onboard the International Space Station (ISS) in the 2020-2025 timeframe. Improvement is expected to come from the accuracy of the clock (at the $10^{-17}$ level in fractional frequency) and the better performance of the time/frequency transfer links. Many new experiments related to fundamental physics in space are described by Delva et al. (2017). This is a very active field that relies and thrives on technological developments, in particular of clocks, long distance radio and optical links, and inertial sensors (e.g., accelerometers and gyroscopes).

\section{Role of Clocks in Fundamental Geodesy}

Atomic clocks are also outstanding tools for experiments and observations performed in fundamental geodesy such as VLBI (Very Long Baseline Interferometry), pulsar timing, navigation, etc. Clocks are currently used for this purpose on Earth. However, precise measurements from space with a relay satellite are foreseen where clocks will play a fundamental role.

One of the missions that is expected in the future is the E-GRASP/Eratosthenes mission, a present proposal for the ESA's Earth Explorer Mission EE-9. E-GRASP/Eratosthenes is dedicated to improving and homogenizing time and space references on Earth and, more directly, to realizing the terrestrial reference system with an accuracy of $1 \mathrm{~mm}$ and a long-term stability of $0.1 \mathrm{~mm} /$ year, which have so far not yet been achieved. The EGRASP/Eratosthenes platform assembles all the geodetic techniques used for the realizations of reference frames and timescales, themselves used in many high-accuracy geophysical applications. For instance, quantifying sea-level change or the effects of ice melting using altimetry strongly depends on an accurate determination of the position of continental or island reference stations, such as those located at tide gauges, as well as the ground stations of the tracking networks. Previous terrestrial reference systems have been realized by a 
combination of the four space geodetic techniques (VLBI, GNSS (Global Navigation Satellite Systems), DORIS (Doppler Orbitography and Radio-positioning Integrated by Satellite), and SLR/LLR (Satellite and Lunar Laser Ranging)) to optimally use the strengths of each technique. The Global Geodetic Observing System (GGOS) was launched by the International Association of Geodesy (IAG) with the goal of providing a consistent high-quality reference frame for observing changes in the Earth system. The documents delivered by IAG demonstrate the need for the above-mentioned accuracies. This requirement is especially driven by the fact that stability in the present global terrestrial reference frame (TRF) of about $0.5 \mathrm{~mm} /$ year is one of the major uncertainties that is mapped in the determination of global sea level rise. The importance of a highly accurate, stable and sustainable reference frame has even been recognized by the A/RES/69/266 United Nations Resolution ${ }^{1}$. The full and rigorous combination of all space geodetic techniques rely on including the common clock of high precision in space. The mission is undergoing a down-selection at present for the future EE9 Explorer Mission. Even if the mission is not selected, the scientific goals remain of high importance for the scientific community as well as for the society.

It is worth mentioning that high-precision clocks in the context of the realization of reference frames and time scales used in geodesy as mentioned above, will also soon require the re-definition of time scales or, at least, their clarification to ensure their continuity and consistent use in practice, as discussed by another paper of this topical collection (Müller et al. 2017).

Let us come back to the highly precise time and stable reference frequencies in the frame of space geodesy techniques themselves. It is a fundamental requirement that VLBI ground stations or the GNSS and DORIS satellites are equipped with high-precision clocks. The precision of these clocks evolves with time, and their number used in the satellites increases as well. In particular, the new generation of GPS satellites are equipped with cesium and rubidium clocks/atomic frequency standards. The Galileo satellites carry four atomic clocks each to ensure strong, quadruple redundancy of the timing subsystem: two Rubidium Atomic Frequency Standard (RAFS) clocks and two Passive Hydrogen Maser (PHM) clocks. It has recently been reported that anomalies have been noted in the atomic clocks serving Europe's Galileo satellites. A few RAFS \& PHM clocks unexpectedly failed showing consistent signatures, linked to probable short circuits or long turn-off periods, which is presently under investigation. The only implemented to date example of an Active Hydrogen Maser aboard a scientific satellite mission is the Space VLBI mission RadioAstron/Spektr-R, which has demonstrated promising preliminary results in the gravitational redshift experiment aimed at verification of the Einstein Equivalence Principle (see D.A. Litvinov et al., Phys. Lett. A (2017), in press, arXiv:1710.10074).

Satellite Laser Ranging (SLR) is another geodesic technique, which differs from all other applications like VLBI, GNSS DORIS by the fact that it is a two-way transfer technique. That means that there is no need for a clock synchronization process between both ends of the distance covered by the measurement technique. The paper of Schreiber and Kodet (2017) in this topical collection discusses the role of time in SLR and time transfer. In particular, laser optical techniques for optical clocks over distances of thousands of $\mathrm{km}$ and for time transfer are discussed and shown to be of high importance. Also very promising, although not discussed in the paper, is free-space optical telecommunication. On Earth, this optical communication technology uses light propagating in free space to wirelessly transmit data for telecommunications or computer networking. It is presently being studied also for interplanetary links. We believe that new emerging technologies, and in particular those

\footnotetext{
${ }^{1}$ http://www.un.org/en/ga/search/view_doc.asp?symbol=A/RES/69/266
} 
associated with optical links such as Interplanetary Laser Ranging (ILR) - addressed by the topical collection paper of Dehant et al. (2017), active and passive retro-reflectors, Light Detection And Ranging (LIDAR) can overcome the limitations of radio technology and improve the measurements' precision significantly.

Very Long Baseline Interferometry is a radio astronomy technique developed for observations of celestial radio sources. It is used to study these sources as well as realize the celestial reference frames. Currently, hydrogen maser clocks are used as a standard ingredient of VLBI instrumentation enabling coherent processing of radio signal of celestial sources received at widely separated radio telescopes. Improving the quality of VLBI observations especially at $\mathrm{mm}$ - and sub-mm-wavelengths would require getting frequency stability of local oscillators beyond achievable presently by Hydrogen maser frequency standards.

\section{Role of Clocks in Gravity Field Determination}

As explained above and detailed in this topical collection by Müller et al. (2017), time measured by an ideal clock, when compared to another distant clock, crucially depends on the gravitational potential and velocity of the clock according to general relativity. As clock precision is now at the level of $10^{-18}$ in fractional frequency, they are sensitive to very small differences of the gravitational field, which makes them very useful for understanding the relativistic gravitational physics. According to general relativity, the relative frequency shift between two clocks is directly related to the difference in the values of the gravity potential at the positions of the clocks. This can be directly used to infer gravitational potential differences between different locations as described by Müller et al. (2017). Indeed, a relative accuracy of clocks of $10^{-18}$ is equivalent to measuring the gravitational red shift effect between two clocks corresponding to a height difference amounting to $1 \mathrm{~cm}$. Applying this conceptually new approach can facilitate or even fully resolve the problem of the systematic discrepancies existing between various height systems on the regional, national, and intercontinental levels (known to reach centimeters to decimeters). Müller et al. (2017) conclude that atomic clocks and time metrology will provide us with unique opportunities to support and largely extend the tools for gravity field measurements and physical height determination based on the direct access to the gravity potential differences.

\section{Role of Clocks in Fundamental Astronomy}

Timing has always been key for astronomers. The issue of data timing is fundamental for observations, whatever wavelength (visible, ultraviolet, infrared...) they are at. This is particularly true for the ongoing mission Gaia. With the objective of providing a highly stable and accurate time tag for each observation, the Gaia spacecraft has a Rubidium clock onboard, which is regularly synchronized with the aid of clocks on Earth. The procedure is described by Klioner et al. (2017), taking into account all known effects (e.g., relativity, tropospheric delay, etc.).

Furthermore, optical detection of molecules with large-dish ground-based telescopes or the detection with satellite-based spectrometers need accurate clocks as well. In particular, it is important for searching for variation of fundamental constants from astronomical observation of molecules, which is the subject of the paper by Ubachs (2017). The status of searches for varying constants from astronomical observation of molecules is reviewed, focusing on the dimensionless proton/electron mass ratio. Improvements, currently established 
on some telescope-based spectrographs, focus on the use of frequency comb lasers (a laser source whose spectrum consists of a series of discrete, equally spaced frequency lines) at the observatories. Frequency comb lasers deliver a very dense spectrum of frequency markers at a separation governed exactly by an atomic clock, thus constituting an ideal reference spectrum.

Particular attention is given to pulsar timing, as pulsars are natural cosmic clocks. On long timescales, they could rival the precision of terrestrial atomic clocks. Since pulsars provide a time-standard that is completely independently from the conditions on Earth or the Solar System as a whole, they serve as an interesting complementary tool to atomic clocks. Efforts are indeed on-going to establish such an independent time standard based on pulsars. At the same time, the clockwise behavior of pulsars also serves as an invaluable tool for precision tests of gravity under strong-field conditions and for strongly self-gravitating bodies. Here, binary pulsars test complement information available from gravitational wave detectors, and also provide stringent constraints on alternative theories of gravity. In the near future, an array of observed pulsars will allow the detection of low-frequency gravitational waves, extending the observable frequency range down to $\mathrm{nHz}$-frequencies. Finally, using measurement of pulse arrival times also allows a number of applications for navigation and gravitational wave detection as described by Becker et al. (2017).

\section{Role of Clocks in Planetary Science}

For planetary science, accurate clocks are mainly used as part of an onboard radioscience transponder, either onboard orbiters or rovers or landers at the surfaces of planets or moons. There are several ways of implementing the radio link between a spacecraft and the Earth or between two spacecraft, as discussed by Dehant et al. (2017). In practice, one can do a direct one-way link or a two-way link (outward and return) or even three-way link (involving a triangular link). The two-way radio link is the dominant type for planetary radioscience, as an accurate spacecraft clock is not necessary onboard the spacecraft since the measurements can be calibrated using high-precision clocks on Earth. In the case of one-way radio data, however, the accuracy of the measurements depends on the accuracy of the onboard clock. For reasons of mass and power saving, the satellite clock is usually a simple ultra-stable oscillator, and thus, large equipments required for atomic clocks are not necessary.

However, the possibility of using high-performance clocks with accuracy of $10^{-14}$ or better in space may change the picture significantly. In particular, the special case of using laser ranging and other techniques to the Moon and the implication of having an accurate clock on the Moon is shown as an example of high-level science by Dehant et al. (2017).

\section{Perspectives and Conclusion}

2018 will see changes in the definition of units, which, in due course, will all rely on time and frequency standards, as time is by far the most accurately measured fundamental physical quantity. Without a doubt, future science and many applications on Earth will rely more and more on highly accurate atomic clocks. Complementary future advances in space science like geodesy, astronomy, and planetary science will follow suit, with the first and probably most demanding application being fundamental physics on ground and in space. 